\title{
THE LOW-FIELD CONDUCTIVITY OF ZEOLITE-ENCAPSULATED MOLECULAR WIRES
}

\section{ZUPPIROLI}

Laboratoire de Physique des Solides Semi-cristallins, IGA-DP, EPFL, CH-1015 Lausanne

F. BEUNEU and J. MORY

Laboratoire des Solides Irradiés, Ecole Polytechnique, 91128 Palaiseau Cedex (France)

P. ENZEL and T. BEIN

Department of Chemistry, Purdue University West Lafayette, Indiana 47907 (USA)

\begin{abstract}
The first measurements of an upper bound for the low-field conductivity of a molecular wire are presented here. We were able to encapsulate polypyrrole with chain lengths more than 10 monomers within the channels of different zeolites. Although the chains are fully oxidized by intrazeolite $\mathrm{Fe}^{3+}$ ions, and should conduct (when included in a bulk polymer), they do not exhibit, in the zeolite, significant ac conductivity up to $1 \mathrm{GHz}$. This suggests that other strategies than low field conductivity are needed to inject charges and transmit information through isolated molecular wires.
\end{abstract}

In common biological processes information is transmitted and processed at the molecular scale, while the best artificial integrated circuits (memories) achieve only a scale of about $1 \mu \mathrm{m}$. There has been substantial interest in the design of "molecular electronic" devices based on molecular units. This concept poses enormous challenges, including the problem of access to molecule-sized structures, defects and heat dissipation. Moreover the physical aspects of electron-transfer at the molecular scale deserve further investigation. In a project aimed at the understanding and design of conducting nanostructures, we have recently succeeded in the preparation of zeolite-encapsulated chains of polypyrrole [1], polythiophene, and other conjugated polymers. The first measurements of an upper bound for the low-field conductivity of a molecular wire are presented here.

During the past twenty years, research in the field of organic conductors has resulted in many "molecular wires", in the form of stacks or bundles of polymer chains. However, these species are always associated in crystals, fibers or films, which causes electronic interchain coupling. These interactions are often weak but nevertheless determine most of the bulk electronic properties.

Signals in biological systems are usually transmitted through membranes or other organized structures, in order to limit interference and to spatially separate events from each other. We have developed systems that mimic the stabilizing function of membranes by encapsulating conjugated polymers in an inorganic host with nanometer size ordered pores: a molecular sieve [1]. 
The two zeolites have been used in the present study: Faujasite (FAU) consisting basically of a diamond cubic lattice of $1.2 \mathrm{~nm}$ cavities connected by $0.75 \mathrm{~nm}$ windows, and mordenite (MOR) consisting of an assembly of $0.7 \mathrm{~nm}$ wide, parallel channels.

Electrochemical studies of the pyrrole polymerization have shown that, in order to obtain polypyrrole in the fully oxidized conducting form, the capture of 2.3 electrons per pyrrole monomer is required on average. Two of the electrons are used for oxidative ring coupling and the remaining 0.3 electrons captured at the electrode generate predominantly hole-type carriers in the form of spinless bipolarons, or spin excitations in the form of polarons [2]. In order to transfer this synthesis into the zeolite, oxidizing species have to be provided in the zeolite interior: these are $\mathrm{Fe}^{3+}$ ions introduced by ion exchange in water solution and successive oxidation (FAU: $10 \mathrm{Fe}^{3+} / \mathrm{unit}$ cell; MOR: $2.5 \mathrm{Fe}^{3+} / \mathrm{unit}$ cell). After complete dehydration of the zeolite channels (at $400^{\circ} \mathrm{C}, 10^{-5}$ torr), the pyrrole monomer is introduced from a solution of hexane under exclusion of air, and polymerization is completed after about one hour at $25^{\circ} \mathrm{C}$. Because monomer adsorption is fast compared to polymerization [1], accumulation of polymer in the outer regions of the crystal is unlikely. Indeed, electron micrographs did not show any accumulation of polymer material on the outer zeolite crystal surfaces.

In order to characterize the encapsulated chains properly, it is important to answer the following questions:

- What is the oxidation state of the polymer,

- what is the nature of the charge carriers,

- how long are the chains on average?

The first two questions were resolved with electron spin resonance experiments (Bruker ER 200D), and information about chain lengths was obtained from optical absorption spectra (Hitachi U4001, with integrating sphere). The characterization of the intrazeolite systems was assisted by previous ESR in situ experiments by Genoud et al. [3], optical spectra by Patil et al. [2], and recent ESR and optical measurements of Caspar et al. [4] on intrazeolite thiophene oligomers.

It is important to summarize first the major feature of bulk polypyrrole films. These films are typically produced electrochemically at the anode of a cell containing a solution of pyrrole (Py), and, for example, perchlorate ions. The overall electrode reaction can be expressed as:

$3 n \mathrm{Py} \rightarrow 7 \mathrm{n} \mathrm{e}^{-}+6 \mathrm{n} \mathrm{H}^{+}+\left[\left(\mathrm{Py}_{3}\right)^{+}\right]_{n}$

The direct result of this electropolymerization is always a fully oxidized polymer which is conducting because it contains holes in excess. The polymer charge is compensated by the $\mathrm{CIO}_{4}$ ions entering the film. The oxidized polymer can be reduced by reversing the cell potential. The charge carriers in these systems are not common band holes but fairly localized singularities of the electron density gliding along the chains with low activation energy. Depending upon the degree of oxidation, the singularities may be described as cation radicals (polarons) or spinless dications (bipolarons). The oxidation state of the intrazeolite polymer chains can thus be deduced from the spin density obtained in ESR experiments. 


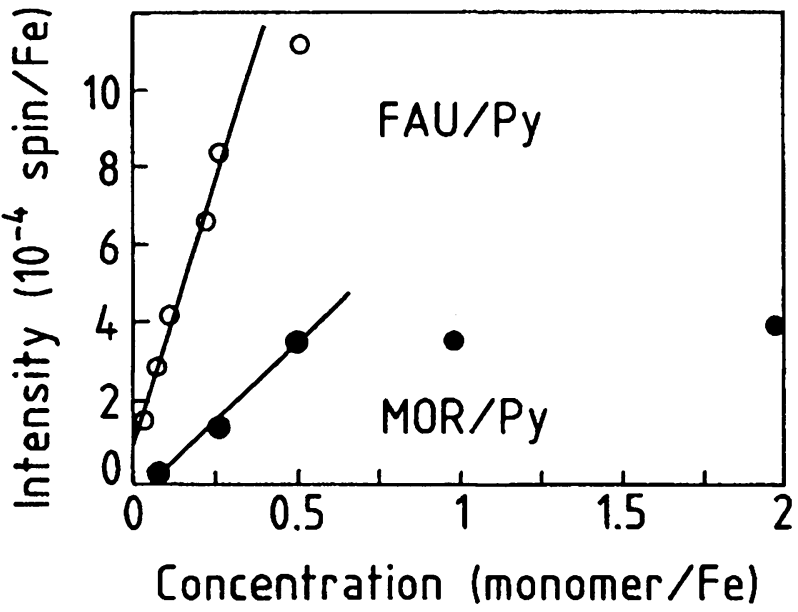

Fig. 1. Spin concentration of polypyrrole encapsulated in faujasite (FAU) and mordenite (MOR) as a function of monomer concentration.

The ESR spectra show two lines that are both expected: one at $g=2.003$ represents the polymer [3], the other at $\mathrm{g}=4.28$ represents the paramagnetic $\mathrm{Fe}^{3+}$ ions in a non-cubic environment [5]. Both signals originate from non-interacting spins because the signal intensities follow accurate Curie laws between $3 \mathrm{~K}$ and room temperature. While the oxidation of the monomer proceeds, the iron signal decreases and the polymer signal intensity increases.

In Figure 1, the number of polymer spins is plotted as a function of the monomer concentration, given in molecules per $\mathrm{Fe}^{3+}$ oxidizing ion. Independent of the topology of the channels and of the precise distribution of iron ions, the number of spins is proportional to the concentration of monomer used, up to a saturation value lower than 0.5 monomers per oxidizing center. Thus, up to this concentration, all the monomer introduced encounters oxidizing Fe(III) centers in sufficient number and is polymerized, the linear dependence just reflecting the fact that the ratio of polarons to spinless bipolarons is roughly constant along the chains and independent of the total polymer content. The stoichiometry required here is also close to the $3 n$ of the electrochemical process. Even though some of the Fe(III) ions are initially located in small cavities (in FAU), the introduction of organic molecules such as the solvent or monomer is well known [6] to change their site distribution, moving them to the accessible cavities and making the oxidation more facile; in this way, all the iron can be used. In the linear part of the curves, the number of non-interacting spins responsible for the polymer signal are about $2.6 \times 10^{-3}$ per monomer in the faujasite and $1.4 \times 10^{-3}$ in the mordenite. According to reference 3 , this low number of spins could be attributed either to a non-oxidized polymer or to a highly oxidized one. Only the latter solution is valid here because we know from the $\mathrm{g}=4.28 \mathrm{ESR}$ signal that $90 \%$ of the FeIII centers have disappeared. Indeed the redox potential of the $\mathrm{Fe}$ (III)/Fe(II) pair $(+0.77 \mathrm{~V}$ at acidic standard conditions) is comparable to the potential applied in complete 
electrochemical oxidations, and Fe(III) has been used successfully for chemical syntheses of polypyrrole powder and films. thus the intrazeolite conditions permit polymerization and complete oxidation of the adsorbed pyrrole monomer.

How long are the intrazeolite polymer chains? The determination of polypyrrole chain lengths is always indirect, even in bulk samples, because the polymer is insoluble. However, the optical data show in our case, that the polymer chains are much longer than 10 units. In Figure 2, different spectra of chains encapsulated in zeolites are presented and compared to the data of thiophene $\left(9^{++}\right)$ and $\left(6^{++}\right)$oligomers reported in reference 4 . The intrazeolite chains exhibit the same broad absorption from 500 to about $2500 \mathrm{~nm}$ as the bulk [2] and not at all the resolved spectra of well-defined short oligomers. The presence of bulk-like chain length is also confirmed by the insoluble nature of polymers recovered from dissolved zeolite host (in HF or critic acid).

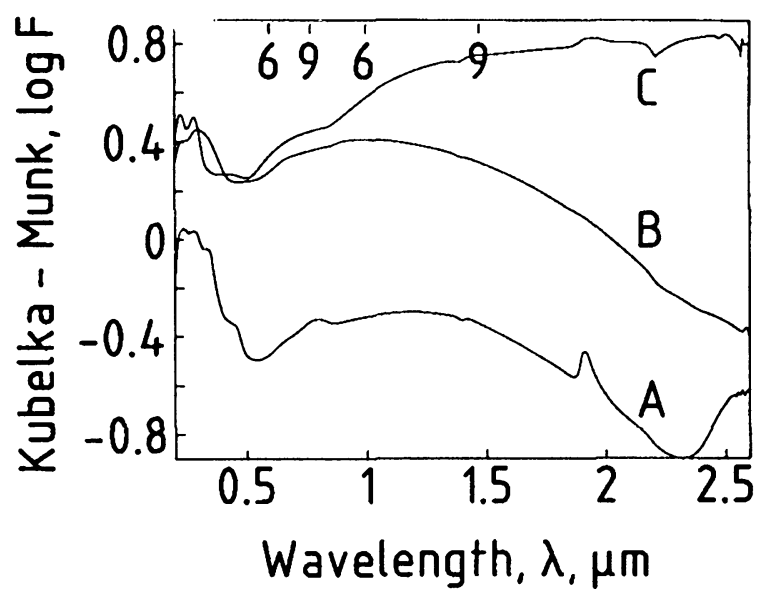

Fig. 2. Reflectance spectra of intrazeolite polymers. (A) Pyrrole in Fe(III) MOR, (B) pyrrole in Fe(III) FAU, (C) tiophene in Cu(II) FAU. The bars indicate the position of the well defined lines of the hexamer $\left(6^{++}\right)$and the nonamer $\left(9^{++}\right)$of tiophene in ZSM-5 zeolite 6 . As in reference 6 , the spectrum is presented in Kubelka-Munk units $\left(F=(1-R)^{2} / 2 R\right)$, where $R$ is the reflectance coefficient.

Are the intrazeolite wires conducting? Bulk polypyrrole with oxidized chains would exhibit low field conductivity of the order of $1(\Omega \mathrm{cm})^{-1}$. We have not yet attempted to attach macroscopic contacts to single polymer chains. Instead, contactless microwave (a.c.) absorption measurements were performed in the range $100 \mathrm{MHz}-1 \mathrm{GHz}$. Even interrupted polymer wires would be detected in this experiment by a significant increase of the dielectric constant with respect to a reference sample containing only monomer molecules. 


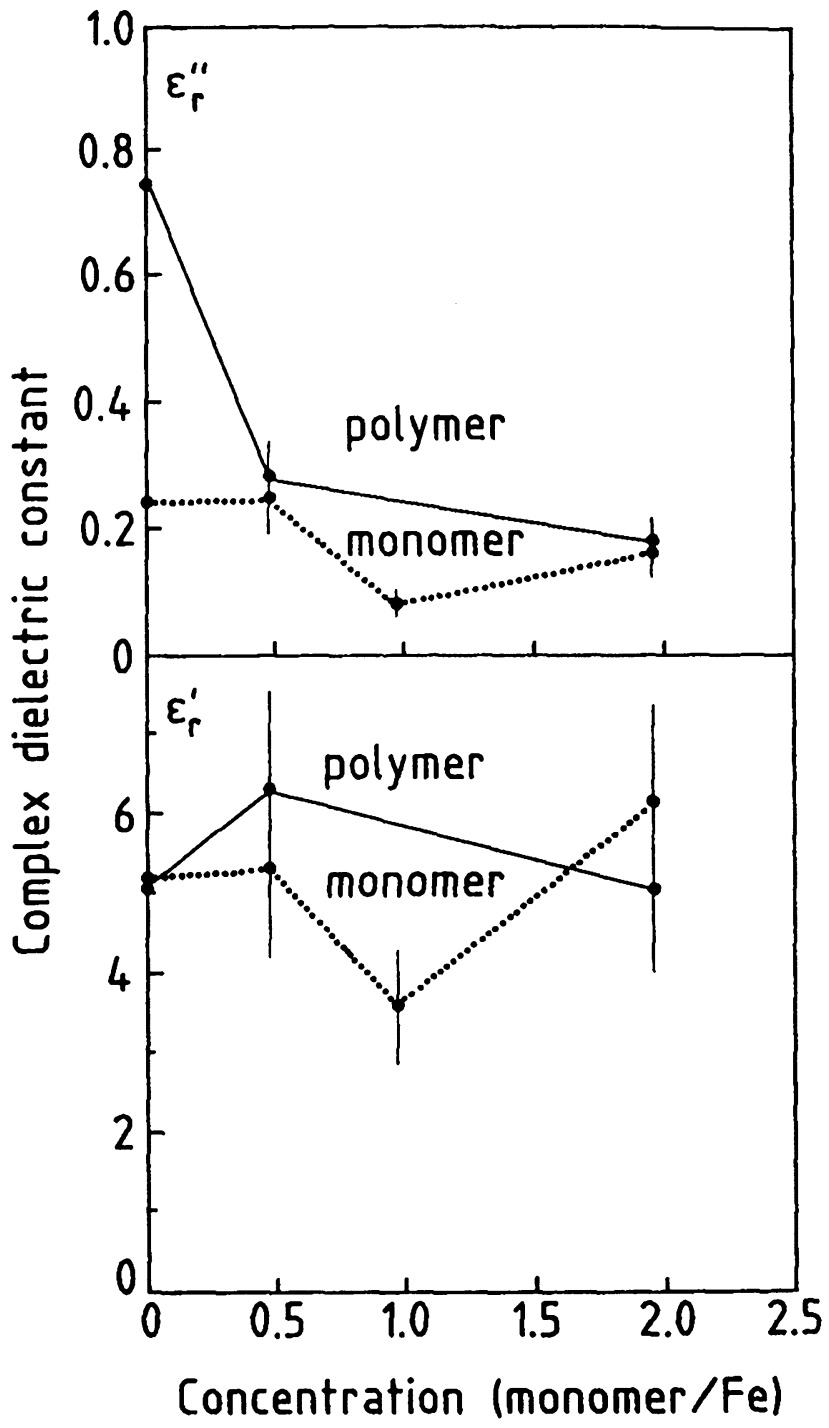

Fig. 3. Dielectric constant $\left(\varepsilon^{\prime}\right)$ and dielectric loss $\left(\varepsilon^{\prime \prime}\right)$ of intrazeolite pyrrole as a function of the monomer concentration. We compare at identical concentrations a sample in which the pyrrole is polymerized to a sample in which it is not. In both cases, the matrix is eventually the same faujasite with $\mathrm{Fe}^{2+}$ ions.

Sample for the a.c. measurements were immersed in wax and molded into a toroidal shape appropriate for accurate determination of the dielectric constants. The presence of a wax coating around the zeolite grains required a deconvolution of the complex dielectric constant measurements according to the Maxwell-Garnett formula [7]. Within the error bar of $20 \%$ on the final results, the real $\left(\varepsilon^{\prime}\right)$ as well as the imaginary part $\left(\varepsilon^{\prime \prime}\right)$ of the relative dielectric constant is frequency independent 
between $100 \mathrm{MHz}$ and $1 \mathrm{GHz}$. To check the validity of this deconvolution, we have directly measured the zeolite powder in a cavity at selected higher frequencies, finding absolutely consistent results. Representative values at $500 \mathrm{MHz}$ are plotted in Figure 3 as a function of the monomer concentration c. The fully oxidized polypyrrole samples in the zeolite host are compared to a $\mathrm{Fe}^{2+}$ non-oxidizing zeolite of the same structure containing the same amount of monomer. The complex behaviour reported in Figure 3 is the result of several contributions to the dielectric relaxation: ions in the host structure, polarizability of the monomer, and possibly conduction. Assuming that the difference between the value of $\varepsilon^{\prime \prime}$ for the polymer and the monomer samples has to be entirely attributed to the conductivity $\sigma$ in the polymer chain, one obtains a value for $\sigma$ of about $5 \times 10^{-6}(\Omega . \mathrm{cm})^{-1}$ at the lowest measurement frequency of $100 \mathrm{MHz}$. This upper bound of the conductivity is very low, even when one takes into account the fact that the polymer is (at the higher concentrations) about five times less dense in the zeolites than in the bulk. If we believe that this low value is due to interruptions in the chains without any significant possibility of tunneling through the barriers, then actual conduction should cause higher values of the real dielectric constant $\left(\varepsilon^{\prime}\right)$ at the order of a few ten (at least), which is obviously not observed. The similar behaviour of polypyrrole chains in very different zeolite hosts (three-dimensional FAU and one-dimensional MOR) demonstrates that the detailed structural definition within the channels is not relevant here. After complete dissolution of the zeolite in HF, we were not able to measure the AC conductivity at $100 \mathrm{MHz}$, but the $\mathrm{DC}$ conductivity of the residue was found to be larger than $10^{-4}(\Omega-\mathrm{cm})^{-1}$. This corresponds to a very significant increase of the conductivity outside of the matrix.

What is the reason for the absence of significant conductivity within the zeolite? The absence of coherent band-conduction in strictly one-dimensional systems has been established very clearly: any significant disorder localizes all the electronic states and any electron-phonon interaction causes selftrapping of the carriers [8]. In both cases conduction can only occur through inelastic processes like hopping. Fortunately, in conducting polymers in general, solitons, polarons, bipolarons in large numbers diffuse along the chains with very low activation energies. This was the reason to chose conducting polymers as potential molecular wires in the present work. The low values of the mobilities that we have found here, demonstrate that, in the present system, polarons and bipolarons are trapped. The ESR line-width of the encapsulated polymer confirms this picture: it decreases monotonously from 11 to 8.5 gauss in modernite and from 9.5 to 6.5 gauss in faujasite with increasing polymer concentration. These values are large with respect to those in clean bulk polypyrrole $(\sim 1 \mathrm{G})$. Similar values could have been obtained in the bulk, by polluting the material by oxygen. They are typical of pinned polarons.

It is useful to compare this result with a recent important study on caroviologens incorporated in vesicle membranes [9]. These conjugated molecules showed significant electron transfer through the membrane as detected by redox reactions with metal complexes. The difference between these two situations results from the different carrier energies in both systems. Microware field excitation of the intrazeolite chains can be described as an attempt to propagate charge carriers that are very close to the Fermi energy level, while redox reactions in the membrane inject the charge carriers far from the Fermi level where the propagation is much easier. 
This study shows that other strategies than low-field conductivity (for example, redox or optical excitations) might be required to transfer charges along conjugated molecular chains in solid host systems as those discussed in this article, and molecular wires will always be supported by a host system in any reasonable application.

\section{ACKNOWLEDGEMENT}

We would like to thank Stephane Esnouf for his contributions to some of the ESR measurements and to the microwave cavity experiments.

\section{REFERENCES}

1 T. Bein and P. Enzel, in Angewandte Chemie, International Ed, in English 28 (1989) 1962.

2 A.O. Patil, A.J. Heeger and F. Wudl, in Chem, Rev. 88 (1984) 183.

3 M. Nechtschein, F. Devreux, F. Genoud, E. Vieil, J. Pernaut and S. Genies, in Synth. Metals. 15 (1986) 59.

4 J.V. Caspar, V. Ramamurthy and D.R. Corbin, in J. Am. Chem. Soc. 113 (1991) 600.

5 T. Caster, G.S. Newell, W.C. Holtan and C.P. Slichter, in J. Chem. Phys. 32 (1960) 668.

6 E. Dendooven, W.J. Mortier and J.B. Vytterhoeven, in J. Phys. Chem. 88 (1984) 1916.

7 R. Landauer, in Electrical Transport and Optical Properties in Inhomogeneous Media. A.I.P. conf. proceedings No 40 (J.C. Garland ed.) (1978) 2.

8 L. Zuppiroli, in Physics of the molecular wire. C.E.A. report R-5543 (1991).

9 T.S. Arrhenius, N. Blanchard-Desse, M. Dvolaitzky, J.J. Lehn and J. Malthete, in Proc. Natl. Acad. Sci. USA 83 (1986) 5355. 